\title{
Interplay between size, morphology, microstructure defects and optoelectronic properties of CdSe nanocrystals
}

\author{
S. Neumann ${ }^{1}$, M. Rudolph ${ }^{1}$, C. Menter ${ }^{2,3}$, A. S. Mahmoud ${ }^{2,3}$, D. Segets ${ }^{4}$, D. Rafaja ${ }^{1}$ \\ ${ }^{1}$ Institute of Materials Science, TU Bergakademie Freiberg, D-09599 Freiberg, Germany \\ ${ }^{2}$ Institute of Particle Technology (LFG), Friedrich-Alexander-Universität Erlangen-Nürnberg (FAU), Germany \\ ${ }^{3}$ Interdisciplinary Center for Functional Particle Systems (FPS), Friedrich-Alexander-Universität Erlangen-Nürnberg (FAU), Germany \\ ${ }^{4}$ Process Technology for Electrochemical Functional Materials and Center for Nanointegration Duisburg-Essen (CENIDE), University Duisburg- \\ Essen, Germany \\ St.Neumann@iww.tu-freiberg.de
}

Cadmium selenide nanocrystals (CdSe NCs) are frequently used in optoelectronic devices, as they possess unique optoelectronic properties that are highly sensitive to their size, shape and microstructure. Due to the high sensitivity of the properties to the microstructure features, the structure and microstructure of CdSe NCs must be controlled precisely during the synthesis. The CdSe NCs crystallize in thermodynamically stable wurtzitic structure (space group $P 6_{3} m c$ ), in metastable zinc blende structure (space group $F \overline{4} 3 \mathrm{~m}$ ) and in a mixture of both structures [1]. As a result, another critical issue of the CdSe NCs synthesis is the control of phase composition and formation of microstructural defects, as both issues affect the optoelectronic properties additionally $[2,3]$.

The aim of this study was to correlate the size and morphology of CdSe NCs with their phase composition and with the formation of microstructure defects, and to explain the effect of the microstructure defects on the optoelectronic properties of the CdSe NCs. The CdSe NCs under study were produced using hot injection at temperatures between $225^{\circ} \mathrm{C}$ and $250^{\circ} \mathrm{C}$. X-ray diffraction and transmission electron microscopy with high resolution revealed that the CdSe NCs have a size between 3 and $10 \mathrm{~nm}$, and crystallize predominantly in the metastable zinc blende crystal structure. While NCs having a size smaller than $4 \mathrm{~nm}$ were practically defect-free, larger particles contained planar defects (stacking faults), which number increased with increasing $\mathrm{NC}$ size. When the planar defects appeared randomly in the interior of the NCs, then they led to an anisotropic broadening of the X-ray diffraction lines as typical for isolated stacking faults [4]. When the planar defects appeared on every second cubic lattice plane $\{111\}$, then they accomplished the transition of the zinc blende structure of CdSe to the thermodynamically stable wurtzitic modification [5]. A combination of XRD measurements and simulations using DIFFaX revealed that the interplanar spacing along the stacking direction apparently depends on the density and ordering of the planar defects. Our approach is discussed together with the approach of Moscheni et al. [6].

In general, the planar defects located in the interior of the CdSe NCs deteriorate their photoluminescence quantum yield. Additional planar defects originate from the oriented attachment of CdSe NCs along the $\{111\}$ crystallographic planes. These defects disturb the crystallographic coherence of attached NCs. Consequently, agglomerated NCs are not recognized as large NCs but as separated NCs both by XRD and by photoluminescence.

[1] Bawendi, M. G., Kortan, A. R., Steigerwald, M. L. \& Brus, L. E. (1989). J. Chem. Phys. 91, 7282.

[2] Viswanatha, R. \& Sarma, D. D. (2009). Chem.: Asian J. 4, 904.

[3] Orfield, N. J., McBride, J. R., Keene, J. D., Davis, L. M. \& Rosenthal, S. J. (2015). ACS Nano 9, 831.

[4] Warren, B. E. (1990). X-ray Diffraction. New York: Dover Publication.

[5] Martin, S., Ullrich, C., Šimek, D., Martin, U. \& Rafaja, D. (2011). J. Appl. Cryst. 44, 779.

[6] Moscheni, D., Bertolotti, F., Piveteau, L., Protesescu, L., Dirin, D. N., Kovalenko, M. V., Cervellino, A., Pedersen, S., Masciocchi, N. A. \& Guagliardi, A. (2018). ACS Nano 12, 12558.

Keywords: X-ray diffraction, transmission electron microscopy, cadmium selenide, nanocrystals, planar defects

The financial support of the German Research Foundation (DFG) for funding the research projects (RA1050/25-1 and SE2526/2-1) within the priority program SPP 2045 "Highly specific and multidimensional fractionation of fine particle systems with technical relevance" is gratefully acknowledged. Furthermore, the authors would like to thank Dr. Christian Schimpf for conducting the XRD experiments and Mrs. Astrid Leuteritz for preparing the TEM specimens. 